Military Technical College Kobry El-Kobbah Cairo, Egypt

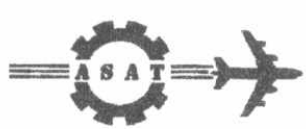

$10^{\text {th }}$ International Conference

On Aerospace Sciences\&

Aviation Technology

\title{
FEATURE EXTRACTION FROM MLAER SIGNALS AND VALIDATION DURING PROPOFOL ANAESTHRSIA
}

\author{
M. Elkfafi*, M.Gadalla*, S. Alian*, T.Soliman*.
}

\begin{abstract}
The depth of anaesthesia (DOA) is generally considered adequate if the patient neither moves in response to surgical stimulus nor shows any signs of autonomic reflexes. So the measuring of depth of anaesthesia is very important because it helps the anesthetist to monitor the anesthetic state of the patient from the start of giving the anaesthetic drugs up to the patient awakeness and heips him to control the required amounts of the anaesthetic drugs during the surgery. There is no direct method to judge the DOA. It seems logical that, since the brain is the primary site of action of anaesthetic agents, there should be some measure of anaesthetic effects on the brain. The brain electrical activity (Electroencephalogram (EEG) and evoked potentials (EP)) have been studied to develop quantitative monitors of DOA. Due to the large problems with the EEG monitoring, EP monitoring has been suggested as an alternative indicator of anaesthetic depth. The mid latency auditory evoked response (MLAER) was seemed to be the most promising measure of DOA, which is independent of the agent being used.
\end{abstract}

The aim of this paper is to propose an approach to explore a reliable index reflecting the changes in amplitudes and latencies of MLAER waves, which are related to depth of anaesthesia

\section{KEY WORDS}

Depth of Anaesthesia (DOA), Feature Extraction (FE), Evoked Potentials (EP), Mid Latency Auditory Evoked Response (MLAER), Central Nervous System (CNS), Signal to Noise ratio (S/N), Fast Fourier Transform (FFT), Auditory Evoked Response (AER), Propofol Concentration (PC), Digital Signal Processing (DSP), Averaged Phase of MLAER signal in S - domain (Ph), Averaged Magnitude of MLAER signal in $S$ - domain (Mag).

\footnotetext{
*Egyptian Armed Forces.
} 


\section{I) Introduction}

General anaesthesia implies the loss of all sensation throughout the whole body and is associated with unconsciousness. General anaesthesia is defined as irregular descending depression of the nervous system, and it is also defined as a state in which certain physiological systems of the body are brought under external regulation by the action of various chemical agents (Collins, 1993).

A sensory evoked potential (EP) is the electrical activity that is elicited in a nervous pathway by a sensory stimulus [Kakigi, 1994]. When a sensory stimulus such as an auditory click or a light flash is applied to a subject, information about the stimulus is translated by the relevant sensory organ into a sequence of action potentials, which are then transmitted through a nervous pathway to the central nervous system (CNS).

Monitoring of DOA is essential and challenge anaesthetists to prevent patient's perception of pain, awareness, and recall; to judge autonomic status and prevent untoward effects of it excessively light or deep anaesthesia; to minimize stress and its manifestation; and to facilitate prompt emergence when indicated. Also a good measuring of DOA provides patient safety, small recovery time, no vomiting. It also decreases the use of intensive care units for long time and saves drugs.

Any signal used to monitor the depth of anesthesia should satisfy the following requirements:

(a) Graded changes with anaesthetic concentration.

(b) Similar changes for different agents.

(c) Appropriate changes with surgical events.

(d) Indication for awareness or Very light anaesthesia.

The Auditory evoked response (AER) has been tested with above criteria in mind. Thus having validated the mid-latency auditory evoked response (MLAER) as a suitable measure of depth of anaesthesia [Thronton and Newton, 1989]. The main features of MLAER, which reflect the anaesthetic depth, are the changes in latencies and amplitude of its waves.

\section{II) Digital Signal Processing of MLAER}

In this work, The system processes the raw AER signal, which were recorded from patient during anaesthesia with propofol. The conventional averaging technique is applied to the recorded raw data for small number of sweeps (192 sweeps) to enhance the AER signal. The averaged signal passed through moving average filter, which is suitable for medical processing to smooth it. The length of the moving average filter window for moving average filter is 5 samples. The output signal [MLAER] from this system is enhariced and smoothed with a good signal to noise ratio. [M. Eikfafi., et al., 2003, (in press)]. 


\section{III) Feature Extracting from MLAER}

To extract the basic features that represent the MLAER signals well, the Fourier Transform is applied. Because of the nonstationarity of the MLAER signals, they are framed by applying a rectangular window of length $(16-85 \mathrm{~ms})$. Then the Fast Fourier Transform (FFT) is applied to these windows as given in Equation (1).

$$
Y(k)=\sum_{n=1}^{N} x(n)^{*} \exp \left(-j^{*} 2^{*} p i^{*}(k-1)^{*}(n-1) / N\right), 1 \leq k \leq N .
$$

where,

$x(n)$ is the input MLAER window,

$Y(K)$ is the Fourier transform of $x$,

And $\mathrm{N}$ is the window length (the number of samples).

The MLAER contains 3-positive waves $\left(P_{a}, P_{b}, P_{c}\right)$. After FFT the amplitudes (magnitudes) and phase of these waves are considered to be the main parameters for measuring the anaesthetic depth. The appearance of the 3-waves indicates inadequate anaesthsia (i.e. awareness) while the appearance of the 2-waves $\left(P_{a}, P_{b}\right)$ indicates moderate anaesthesia. The further depression of the wave $P_{a}$ (i.e. more decrease in amplitude and decrease in phase) indicates surgical anaesthesia while flat MLAER (i.e. the 3-positive waves disappear) indicates a deeper level of anaesthesia than is necessary [M. Elkfafi, 1995]. To observe the changes in the 3positive waves, the AER window for analysis was chosen to be $16-85 \mathrm{~ms}$. It was found experimentally that in the model of FE shown in Fig. (1), the sum of the components of the amplitudes (Mag) response of the signal and the sum of the phase $(\mathrm{Ph})$ response describe the changes in amplitudes and latencies of the MLAER wave relating to the DOA.

The above two factors are calculated by the following procedure:

1) Compute the actual output, of the signal averaging of 192 sweeps (i.e. 30 Seconds) after the start of the induction phase and filtering technique.

2) Transform the averaged and filtered signal into the frequency domain using FFT transformation.

3) Calculate the magnitude (Mag) and the phase $(\mathrm{Ph})$ every 30 -second as described in equation (2) and equation (3).

$$
\begin{gathered}
\text { Mag }=\sum_{i=1}^{N}|X|(i) \\
P h=\sum_{i=1}^{N|\Phi|}(i)
\end{gathered}
$$

Where $\mathrm{N}$ is the number of samples, $\mathrm{N}=1,2,3, \ldots \ldots, 70$ 


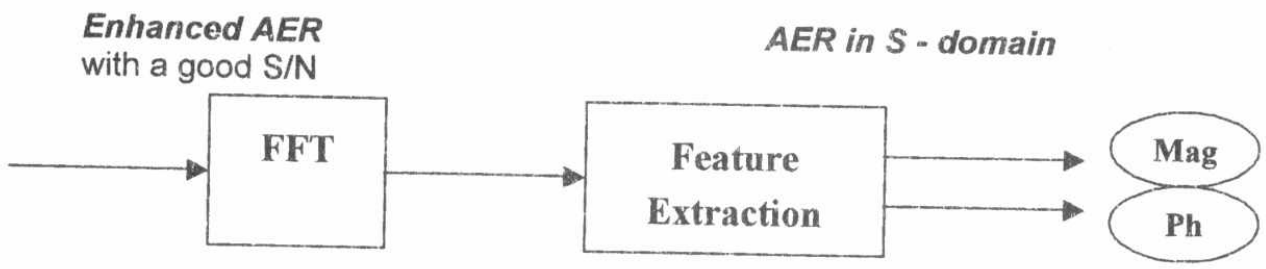

Fig. 1. The structure of the system for FE of AER

\section{IV) Validation of The Extracted Features}

From the literature review described before we are satisfied that the MLAER shows promise for measuring the depth of anaesthesia. To clinically evaluate the tow factors (Mag., Ph.) derived from the MLAER the AER data were recorded from 21 patients, who were undergoing body surface surgery, during intravenous anaesthesia via propofol in the Royal Hallamshire Hospital, Sheffield. At the same time, the propofol concentration (PC) was measured one minute before stopping of propofol and every minute after the stopping of propofol (i.e. during the recovery phase). The AER data corresponding to the period of measuring the PC were selected from the AER data files of the 21 patients. These AER data were processed using the digital signal processing technique [M. Elkfafi et al., 2003,(in press)]. The numerical indices (Mag., $\mathrm{Ph}$ ) over the window 16-85 ms, which includes the MLAER, were derived every 30 second. These indices were used to facilitate racking the changes in the MLAER according to the changes in propofol concentration during time recovery phase. The tow indices and PC were plotted versus the recovery time as shown figures(2) and (3).

The changes in the above two factors Mag., and Ph., with different PC., during the recovery phase were examined. It was found that the Mag., and $\mathrm{Ph}$., values were increasing when the PC values were decreasing. This confirm that the Mag., and Ph., reflected the change in magnitude and latencies of the MLAER during anaesthesia, the two factors detected the awareness (i.e. eye opening) successfully as shown in Fig (2), and Fig. (3), and the anaesthetist experiments for detecting the awareness using the clinical signs.

\section{V) Conclusion:}

In this work, two features have been extracted from MLAER to be used for DOA measurement. Namely, the two features are the magnitude and phase spectrum. The variations of these two features have been validated with the drug concentration in the blood, which recorded during the recovery time of the patient. This evaluation has shown to be promising. 


\section{VI) References:}

(1) Collins, V.J, Principle of anesthesiology- general and regional anesthesia, $3 \mathrm{rd}$ ed., Vol. 1, Lea \&Febiger, Philadelphia, (1993).

(2) Thronton, C., and Newton, D. E. F., The auditory evoked response: a measure of depth of anaesthesia, Balliere's Clin. Anaesth., 3: 559-585,(1989).

(3) M. Elkfafi, S. Elian, M.Gadalla, T.Soliman, Digital signal processing for AER signals during anaesthsia, (2003, in press).

(4) M. Elkfafi., intelligent signal processing of evoked potentials for control of anaesthesia, Ph.D. thesis, Department of Automatic Control and System Engineering, University of Sheffield, (1995). 

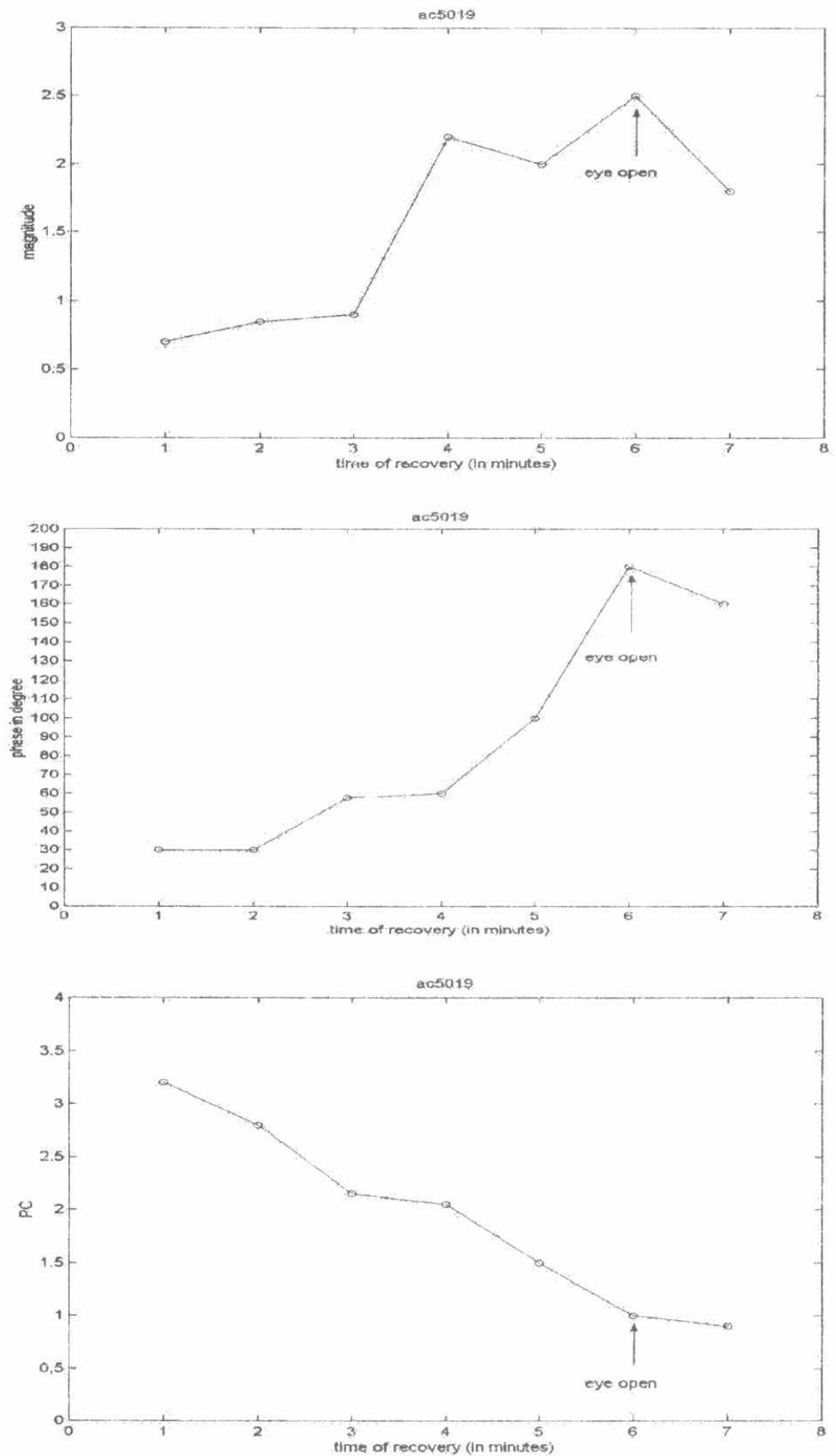

Fig. 2. The changes of Mag., Ph., and PC every one-minute during the recovery phase of the patient AC 5019 

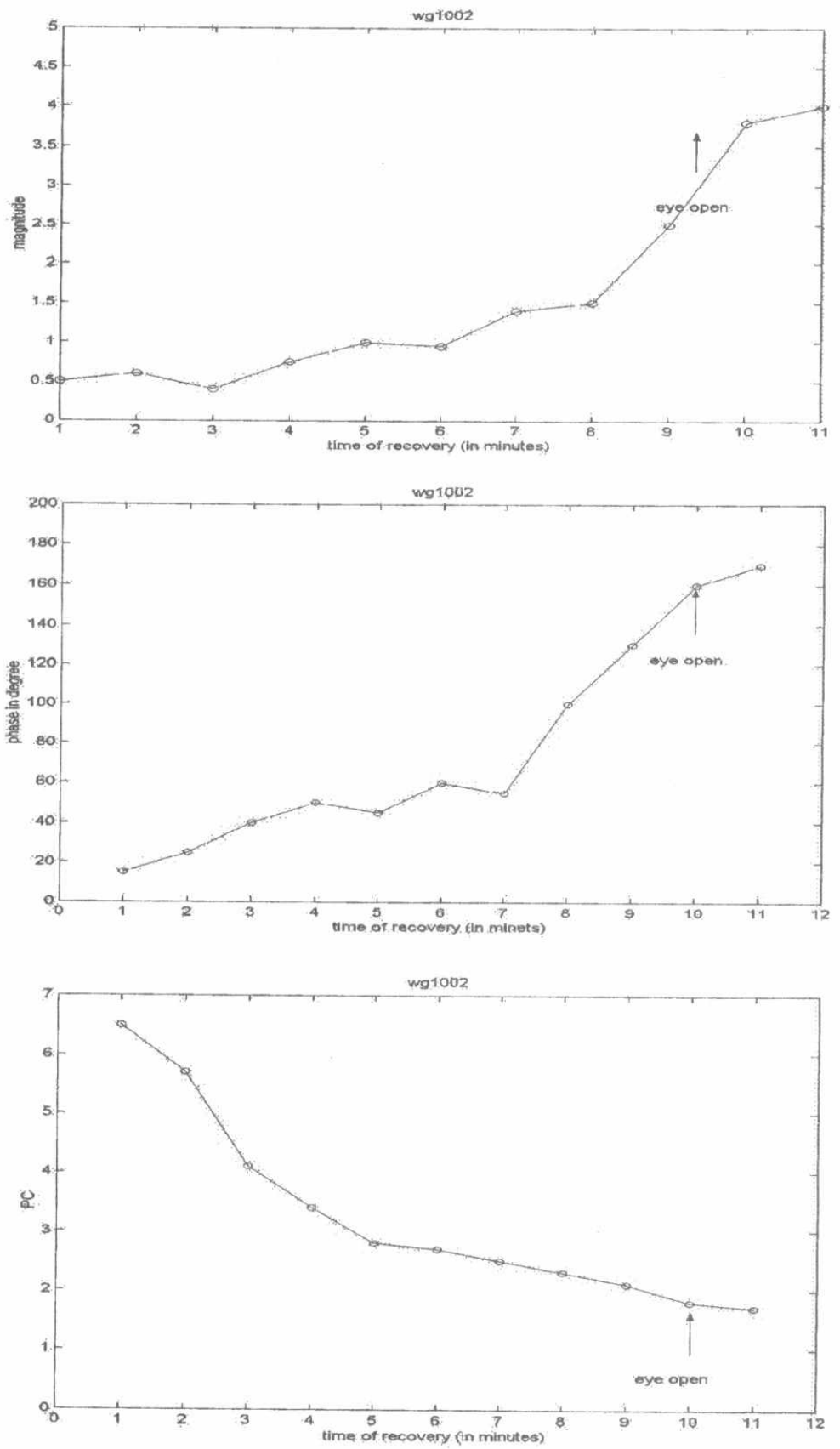

Fig.3 The changes of Mag., Ph., and PC every one-minute during the recovery phase of the patient WG 1002 